\title{
Effectiveness of PhysioDirect telephone assessment and advice services for patients with musculoskeletal problems: pragmatic randomised controlled trial
}

\author{
(c) (1) (8) OPEN ACCESS
}

Chris Salisbury professor of primary healthcare ${ }^{1}$, Alan A Montgomery reader in health services research ${ }^{2}$, Sandra Hollinghurst senior lecturer in health economics ${ }^{1}$, Cherida Hopper trial manager ${ }^{1}$, Annette Bishop research fellow ${ }^{3}$, Angelo Franchini research methods training fellow ${ }^{2}$, Surinder Kaur research associate ${ }^{1}$, Joanna Coast professor of health economics ${ }^{4}$, Jeanette Hall operational lead for outpatient physiotherapy ${ }^{5}$, Sean Grove clinical lead physiotherapy ${ }^{5}$, Nadine E Foster professor of musculoskeletal health in primary care ${ }^{3}$

${ }^{1}$ Centre for Academic Primary Care, School of Social and Community Medicine, University of Bristol, Bristol BS8 2PS, UK; ${ }^{2}$ Bristol Randomised Controlled Trials Collaboration, University of Bristol, UK; ${ }^{3}$ Arthritis Research UK Primary Care Centre, Primary Care Sciences, Keele University, Keele, UK; ${ }^{4} \mathrm{Health}$ Economics Unit, School of Health and Population Sciences, University of Birmingham, Birmingham, UK; ${ }^{5}$ Musculoskeletal Outpatient Department, Bristol Community Health, Bristol, UK

\begin{abstract}
Objectives To assess the clinical effectiveness, effect on waiting times, and patient acceptability of PhysioDirect services in patients with musculoskeletal problems, compared with usual care.

Design Pragmatic randomised controlled trial to assess equivalence in clinical effectiveness. Patients were individually randomised in a 2:1 ratio to PhysioDirect or usual care.

Setting Four physiotherapy services in England.

Participants Adults (aged $\geq 18$ years) referred by general practitioners or self referred for musculoskeletal physiotherapy.

Interventions PhysioDirect services invited patients to telephone a physiotherapist for initial assessment and advice, followed by face-to-face physiotherapy if necessary. Usual care involved patients joining a waiting list for face-to-face treatment.

Main outcome measures Numbers of appointments, waiting time for treatment, and non-attendance rates. Primary outcome was physical health (SF-36v2 physical component score) at six months. Secondary outcomes included four other measures of health outcome, mental component score and scales from the SF-36v2, time lost from work, and patient satisfaction and preference. Participants were not blind to allocation, but outcome data were collected blind to allocation.
\end{abstract}

Results Of 1506 patients allocated to PhysioDirect and 743 to usual care, $85 \%$ provided primary outcome data at six months (1283 and 629 patients, respectively). PhysioDirect patients had fewer face-to-face appointments than usual care patients (mean $1.91 \mathrm{v} 3.11$; incidence rate ratio 0.59 (95\% confidence interval 0.53 to 0.65$)$ ), a shorter waiting time (median 7 days $v 34$ days; arm time ratio $0.32(0.29$ to 0.35$)$ ), and lower rates of non-attendance (incidence rate ratio 0.55 (0.41 to 0.73$)$ ). After six months' follow-up, the SF-36v2 physical component score was equivalent between groups (adjusted difference in means -0.01 (-0.80 to 0.79$)$ ). Health outcome measures suggested a trend towards slightly greater improvement in the PhysioDirect arm at six week follow-up and no difference at six months. There was no difference in time lost from work. PhysioDirect patients were no more satisfied with access to physiotherapy than usual care patients, but had slightly lower satisfaction overall at six months (difference in satisfaction $-3.8 \%(-7.3 \%$ to $-0.3 \%)$; $\mathrm{P}=0.031$ ). PhysioDirect patients were more likely than usual care patients to prefer PhysioDirect in future. No adverse events were detected.

Conclusions PhysioDirect is equally clinically effective compared with usual care, provides faster access to physiotherapy, and seems to be safe. However, it could be associated with slightly lower patient satisfaction.

Trial registration Current Controlled Trials ISRCTN55666618. 


\section{Introduction}

Healthcare systems internationally face the challenge of how to meet increasing demand for healthcare, due to the ageing population and rising consumer expectations within limited resources. Healthcare providers therefore need to explore new approaches to delivering care. One approach that has been used in several contexts is initial assessment by telephone. ${ }^{1}$ This can make it convenient for patients to access advice or treatment (or both), and provides a triage function since patients who have most to gain from early face-to-face treatment can be identified and prioritised. Telephone based approaches have been introduced to assess and advise patients with a wide range of problems in primary care, although have rarely been rigorously evaluated. ${ }^{1}$

This approach could be particularly appropriate for the assessment and treatment of musculoskeletal problems, which are one of the most common reasons for consulting a general practitioner. ${ }^{2}$ Musculoskeletal problems are an important cause of poor health because of pain and functional impairment ${ }^{3}$ and have a major economic effect owing to time lost from work, particularly from back pain. ${ }^{4}$ In the United Kingdom's health service, patients with musculoskeletal problems usually consult in general practice initially. Many are then referred to physiotherapy, with about 1.23 million new referrals from general practitioners per annum in England. ${ }^{5}$ These patients are put on a waiting list to see a physiotherapist for a face-to-face assessment, often followed by a series of follow-up appointments. Providing timely access to physiotherapy has long been a problem in the NHS, with waiting times of several weeks or months for treatment in many areas of the UK. ${ }^{6}$

For some conditions such as neck pain ${ }^{7}$ and shoulder pain, ${ }^{8}$ there is evidence of benefit from manual therapy from a physiotherapist. However, for other conditions such as osteoarthritis, ${ }^{9}$ the main recommended treatment is advice about maintaining physical activities and provision of a structured exercise programme rather than hands on therapy. Therefore, waiting lists might be congested with patients who could benefit from assessment and advice from a physiotherapist, but could have little more to gain from a course of face-to-face appointments.

In an effort to improve access to care, several areas have introduced PhysioDirect services, in which patients can telephone a physiotherapist for initial assessment and advice without waiting for a face-to-face appointment. They can be given advice about self management and exercises over the telephone, and the need and priority for seeing them face-to-face can be established. Services that have implemented PhysioDirect have claimed that it reduces waiting times for treatment, it is popular with patients, and about half of all patients can be managed by telephone alone. ${ }^{10}{ }^{11}$ This type of service is being introduced in several different countries, including the UK, but there is no evidence about health outcomes or costs. ${ }^{12}$ The aim of this study was to assess the clinical effectiveness and cost effectiveness of PhysioDirect compared with usual models of care based on patients joining a waiting list for physiotherapy and eventually receiving face-to-face care. This paper describes the findings with regard to the clinical effectiveness of PhysioDirect, along with findings about access to care and acceptability to patients. The economic analysis is reported elsewhere. ${ }^{13}$

\section{Methods \\ Design}

We conducted a pragmatic, individually randomised controlled trial, incorporating economic evaluation, to compare

PhysioDirect and usual care. ${ }^{14}$ The trial was designed to assess equivalence between the two trial arms in the primary clinical outcome. If equivalence in clinical outcome is established, the parallel economic evaluation and secondary outcomes (such as waiting time for treatment) become particularly important and relevant to future provision of services. The only important change to the study design after it had commenced was an increase in the target number for recruitment, described below.

\section{Setting and participants}

The trial was conducted in four typical community physiotherapy services in four different areas of England (Bristol, Somerset, Stoke-on-Trent, and Cheshire), which drew patients from 94 general practices covering a wide range of types of geographical area and population. Adults (aged $\geq 18$ years) who were referred by general practitioners or who referred themselves for physiotherapy for a musculoskeletal problem were eligible for the trial. Inclusion criteria were deliberately broad to maximise generalisability. The main exclusion criteria were patients with non-musculoskeletal problems, referred by hospital consultants, unable to communicate in English by telephone, who could not be contacted, or with problems deemed (on the basis of the referral form) to be too urgent to be delayed by trial recruitment.

\section{Interventions}

In the UK, usual care typically involves patients with musculoskeletal problems being referred by general practitioners to a physiotherapist. Patients join a waiting list for the next available face-to-face appointment, and could wait for several weeks. After assessment, patients are usually given advice about exercises, supported by advice leaflets. If appropriate, they are invited to a course of further treatment sessions, which might involve hands-on manual therapies.

Patients randomly allocated to the PhysioDirect arm were invited to telephone a senior physiotherapist for initial assessment and advice about treatment as soon as it was convenient.

Physiotherapists were available to receive calls during advertised PhysioDirect sessions. The physiotherapists used computerised templates to assess patients and record findings. As part of their training to provide the PhysioDirect service, physiotherapists were taught enhanced telephone communication skills, including the use of visualisation to help assess patients' symptoms and functional difficulties.

At the end of the call, most patients were sent written advice about self management and exercises and invited to telephone back after two to four weeks to discuss progress. They were then given further advice or offered face-to-face treatment as necessary. The PhysioDirect assessment also made it possible to identify patients who urgently needed face-to-face treatment and other patients who were unlikely to gain benefit from physiotherapy and could be discharged.

The PhysioDirect intervention was based on offering patients an initial telephone assessment with a physiotherapist, with face-to-face care available if necessary. Patients in the PhysioDirect arm could decline a telephone assessment at any time and wait for a face-to-face appointment. The comparison was between a PhysioDirect treatment pathway that offered early telephone advice that, by a system of triage, also allowed 
earlier face-to-face appointments if necessary, and the usual care system in which all patients waited for a face-to-face appointment.

The PhysioDirect services in each of the four sites were standardised as far as possible, with physiotherapists undergoing the same training, ${ }^{15}$ using the same assessment software, and providing the same range of advice leaflets. These advice leaflets were similar to those that may have been given to patients after consultations in the usual care arm.

Computerised assessment templates and training were provided by the physiotherapy team in Huntingdonshire, who have provided a similar PhysioDirect service since $2001 .{ }^{15}$ This study was completely independent, and none of the authors have any conflict of interest in relation to the success (or otherwise) of the intervention. Web appendix 1 provides further details of the PhysioDirect service.

Despite the 2:1 randomisation ratio in favour of PhysioDirect, most patients in each site continued to receive usual care, because it included not only those randomised to usual care but also those ineligible for the trial or declining participation. We allocated physiotherapy staff time in each site in proportion to the number of patients allocated to the PhysioDirect trial arm versus all those due to receive usual care. The time available for patients in the PhysioDirect arm included both telephone sessions and any subsequent face-to-face consultations. Therefore, total physiotherapy staff time per patient was similar in both trial arms. Separate waiting lists were held for patients in the PhysioDirect and usual care arms in each site. By these measures, we ensured that the PhysioDirect service did not affect the waiting time for patients in the usual care arm.

\section{Randomisation and masking}

Patients referred for physiotherapy were invited by post to participate. Those who gave informed consent were randomised in a 2:1 ratio to PhysioDirect or usual care using a secure remote automated allocation system generated and hosted by the Bristol Randomised Controlled Trials Collaboration. We used the 2:1 ratio to ensure that enough patients were randomised to PhysioDirect to make it viable to run PhysioDirect telephone sessions efficiently and at different times in the week. Randomisation was conducted at the level of the patient, stratifying by physiotherapy service and minimising by sex, patient age group, and site of musculoskeletal problem.

Outcomes were self reported in patient questionnaires or obtained by electronic download from the PhysioDirect software or from physiotherapy service records. If patients did not return questionnaires despite reminders, attempts were made to collect primary outcome data by telephone. All outcome data were collected by researchers who were blind to group allocation. It was not possible for physiotherapists delivering the intervention to be blinded.

\section{Outcome measures}

Outcomes were assessed at baseline, and at six weeks and six months after randomisation. We collected data for the process of care including the number, type, and duration of consultations with physiotherapists; waiting times for treatment (defined as first physiotherapy contact); and rates of non-attended appointments.

The primary outcome was clinical outcome at six months, assessed by the physical component summary measure from the SF-36v2 questionnaire. ${ }^{16}$ We used a generic measure because no disease specific measure was appropriate, in view of the wide range of problems experienced by patients in this trial. Despite concerns about the sensitivity of generic measures, the physical component score has been shown to compare well with disease specific measures in patients with musculoskeletal problems. ${ }^{17-20}$ To provide further reassurance that our measures were sufficiently sensitive to detect differences between the groups (particularly important given the equivalence nature of this trial), we also used four other measures of clinical outcome:

- The Measure Yourself Medical Outcomes Profile, a tailored and validated measure that is generated by patients. By focusing on the main problem for which the patient was referred to physiotherapy, this profile would be particularly sensitive to change $\mathrm{e}^{21}$

- The EQ-5D measure of health related quality of life $\mathrm{e}^{22}$

- One question about overall improvement in the main problem for which the patient was referred to physiotherapy (global improvement score—a seven point scale from "very much better" to "very much worse")

- A composite measure of response to treatment including pain, function, and overall improvement as recommended by the Outcomes Measures in Rheumatology Clinical Trials-Osteoarthritis Research Society International initiative ${ }^{23}$

Other secondary outcomes included: the mental component summary score and individual scales from the SF-36v2 questionnaire, time lost from work, patient satisfaction, and patient preference for future care. Questions about satisfaction with the consultation were adapted from the General Practice Assessment Questionnaire, with additional questions about access to the service based on preliminary interviews with patients. We confirmed that these questions formed internally reliable scales using rotated factor analysis. Overall satisfaction with the service was based on one question. All questions used six point Likert scales.

\section{Sample size and power}

The study was powered to establish clinical equivalence in the physical component score, with a difference of no greater than two points conservatively specified as demonstrating equivalence. ${ }^{16}$ Sample sizes for analysis of 976 and 488 patients in the PhysioDirect and usual care groups, respectively, would provide $95 \%$ power to reject a null hypothesis of non-equivalence with an overall two sided $\alpha$ of 0.05 , assuming that the observed difference in means was 0 and standard deviation of the physical component score was $10 .{ }^{16}$

Assuming 20\% non-collection of the primary outcomes, it was necessary to recruit 1875 patients to reach a target of 1500 patients for analysis. Following a protocol amendment, we continued sending invitations until 2000 patients had been randomised, recognising that the final number recruited would be higher because of the lag between invitation and recruitment. This amendment was necessary because of concern about the follow-up rate we would achieve during a national postal strike.

\section{Statistical methods}

We used descriptive statistics to compare characteristics of patients randomised with those eligible but not randomised and to assess the balance between the trial arms at baseline. Analyses of primary and secondary outcomes were conducted on an intention to treat basis without imputation-that is, all patients were analysed according to their randomised groups, except for those who did not provide follow-up data. We used multivariable regression models to investigate between group differences, 
adjusted for stratification and minimisation variables and, if available, the value of the outcome at baseline.

Although the protocol stated that we would make adjustments to $\mathrm{P}$ values arising from analyses of secondary outcomes to account for multiple testing, we later decided not to do so, as discussed in the limitations section of this paper. ${ }^{24}$ In sensitivity analyses, we investigated the effect of any baseline imbalance in outcome variables, clustering by physiotherapy service or general practice, and missing primary outcome data, using multiple imputation methods. ${ }^{25}$ In prespecified subgroup analyses, we explored the effect of PhysioDirect on the primary clinical outcome according to the site of presenting musculoskeletal problem, patient age group, socioeconomic status and physiotherapy service, using interaction terms in the primary regression analysis. All statistical analyses were conducted using Stata version 11.

\section{Investigation of adverse events}

General practitioners and physiotherapists were asked to report any suspected adverse event that may have been related to physiotherapy or to the trial procedures. The general practice notes of each patient were scrutinised by researchers at the end of the trial following a protocol designed to identify any potential adverse events. Data about several other potential harms were available from the main outcome measures described previously, such as continued pain or reduced function (available from scales in the SF-36v2) and extended time off work (from the patient questionnaire).

\section{Trial registration}

The trial was registered with Current Controlled Trials (ISRCTN55666618) before patient recruitment, and the protocol has been published. ${ }^{14}$

\section{Results}

\section{Participant flow and recruitment}

The figure $\Downarrow$ illustrates the flow of patients during the trial. Of 4523 eligible patients, $2256(50 \%)$ agreed to participate. Almost all patients $(98 \% ; 2207 / 2256)$ were referred by general practitioners, the remainder were self referred. Patients who were randomised were slightly younger on average than those who were not (mean age 48 years $v 52$ years), but were otherwise similar in terms of sex, deprivation, site, and referral problem. We recruited and randomised patients between July 2009 and December 2009; 1513 to PhysioDirect and 743 to usual care. Seven patients were found to have been randomised to PhysioDirect in error: two had not completed a valid consent form and five were subsequently found to have exclusion criteria for the trial. We excluded these patients from follow-up and analysis. There were no important differences between groups at baseline (table $1 \Downarrow$ ). Primary outcome data were obtained from $88 \%$ of patients after six weeks' follow-up and $85 \%$ of patients after six months' follow-up.

\section{Process of care}

Of all patients in the PhysioDirect arm, 1281 (85\%) contacted the physiotherapy service at least once. Of the 1237 patients contacting PhysioDirect and assessed initially by telephone, $586(47 \%)$ were managed entirely by telephone. Patients in the PhysioDirect arm had fewer face-to-face appointments than those in the usual care arm (mean $1.91 v 3.11$; adjusted incidence rate ratio 0.59 (95\% confidence interval 0.53 to 0.65$)$ ) and fewer physiotherapy consultations of any type $(2.87 v 3.25,0.87(0.80$ to 0.94$)$ ). Patients allocated to PhysioDirect had a shorter wait for physiotherapy treatment than those allocated to usual care (median 7 days $v 34$ days; adjusted arm time ratio 0.32 (0.29 to 0.35)). Patients in the PhysioDirect arm were also less likely to fail to attend face-to-face appointments over six months than those in the usual care arm (mean failure rate $0.09 v 0.12$, adjusted incidence rate ratio 0.55 (0.41 to 0.73$)$ ).

\section{Primary and secondary outcomes}

PhysioDirect and usual care were equivalent in terms of the primary outcome of physical component score at six months' follow-up (table $2 \Downarrow$ ). This finding was robust to adjustment for baseline imbalance, imputation of missing data, and adjustment for clustering. All measures of health outcome (that is, the primary and four secondary outcomes) showed a similar pattern. These findings suggested a slightly greater improvement in favour of PhysioDirect at six weeks' follow-up, but no differences at six months' follow-up (tables 2 and $3 \Downarrow$ ). However, the differences at six weeks were small and could be clinically unimportant.

Table $4 \Downarrow$ shows no differences between study groups for the mental component summary measure or any of the scales from the SF-36v2 questionnaire. No evidence was found of a difference between the two trial arms in time lost from work (table $5 \Downarrow$ ). Patients in the usual care arm had slightly higher satisfaction than those in the PhysioDirect arm with regards to the consultation and overall satisfaction. No between group difference was evident with regards to satisfaction with access to the service (table $6 \Downarrow$ ).

At baseline, about a third of participants in each arm stated a preference for usual care, stated a preference for PhysioDirect, or expressed indifference. At six months' follow up, 417 (42\%) of 985 patients in the PhysioDirect group and 245 (51\%) of 485 patients in the usual care group preferred usual care; 393 (40\%) and $131(27 \%)$ in each group, respectively, preferred

PhysioDirect. Multinomial logistic regression showed strong evidence that participants in the PhysioDirect arm were more likely than those in the usual care arm to prefer PhysioDirect compared with being indifferent (adjusted relative risk ratio 1.98 (adjusted 95\% confidence interval 1.43 to 1.98 )).

No differences between trial arms in the primary outcome were identified in relation to any of the prespecified subgroups (based on age, referral problem, deprivation, or primary care trust site), but potentially important differences could not be excluded because the confidence intervals were wide (data not shown). No adverse events were detected in either arm of the trial, which included 4323 consultations ( 1445 by telephone) over six months in 1506 patients allocated to PhysioDirect.

\section{Discussion}

Compared with usual care based on waiting lists for face-to-face appointments, a care pathway based on PhysioDirect is equally clinically effective, provides faster access to advice and treatment, and seems to be safe. However, there was no evidence that this pathway was associated with improved patient satisfaction with access to physiotherapy.

\section{Strengths and limitations}

Innovations in the delivery of healthcare are rarely accompanied by large, multicentre, randomised controlled trials such as the present trial, including an analysis of cost effectiveness. ${ }^{26}$ The findings show that randomised trials of service reorganisation are feasible and are also informative in challenging 
assumptions-for example, that faster access to care will reduce patients' time off work or improve satisfaction with the service. However, our study also highlights the limitations of trials of service innovations ${ }^{27}$ For example, providing a new service in the context of a trial meant providing two different care pathways in parallel, which made it difficult to provide PhysioDirect services throughout the week so that patients could call when it suited them. Once the trial ended, far more patients were treated with PhysioDirect by the services that continued to offer it (because there was no consenting process and none were randomised to usual care), and it was possible to provide a more efficient and accessible PhysioDirect service than was tested during the trial.

We observed few differences in outcomes between groups. The differences in clinical outcomes seen after six weeks' follow-up might be too small to be clinically meaningful. However, the pattern of change over time was consistent across several different measures of clinical outcome, supporting the notion that patients allocated to PhysioDirect had a faster improvement in symptoms than those allocated to usual care.

The difference in patient satisfaction was also small and might not be meaningful, with a $3.8 \%$ difference in overall satisfaction, equating to 0.19 points on a six point scale ranging from "very poor" to "excellent." The fact that patients in the PhysioDirect arm (of whom almost half (47\%) were managed entirely by telephone) were almost as satisfied with their consultations as patients in the usual care arm (of whom almost all had face-to-face consultations only) might provide reassurance that physiotherapists are able to provide assessments and advice by telephone in a way that is reasonably acceptable to patients.

The findings about patient satisfaction were also limited because these questions were only answered by people who had had contact with a physiotherapist. By six weeks' follow-up, 50\% of the patients in the usual care arm (compared with $19 \%$ of those in the PhysioDirect arm) had not had any contact. These patients did not complete the questionnaire but may have been those most likely to express dissatisfaction with access.

We did not make adjustments to $P$ values arising from analyses of secondary outcomes to account for multiple testing, which is at variance from the protocol. Our focus was on the magnitude of between arm differences in outcomes using confidence intervals, rather than hypothesis testing using $\mathrm{P}$ values, as a basis for interpretation and decision making. The subject of adjustment for multiple testing has been debated at length by statisticians,${ }^{24}$ and on reflection we decided that the adjustment as stated in the protocol was unnecessary in this case.

The pragmatic nature of this study, which includes a wide range of patients and several physiotherapy sites, makes the findings potentially generalisable to physiotherapy services in the NHS, as well as to the many other countries in which patients experience long delays in accessing physiotherapy. ${ }^{28}$ However, only $50 \%$ of eligible patients agreed to participate. Although this rate of recruitment was high for a community based trial, and no important differences were observed between participants and non-participants, the rate still raises questions about external validity. We do not have data about whether people declined because they did not wish to take part in research or did not want to receive PhysioDirect. However, our recruitment rate suggests that at least 50\% would consider using PhysioDirect if it were offered.

\section{Implications}

This study provides justification for PhysioDirect as a safe and effective way of reducing delays for advice about musculoskeletal problems for patients referred by general practitioners for physiotherapy. But, in view of the slight reduction in patient satisfaction, our results do not provide a compelling argument in favour of PhysioDirect. However, our associated economic analysis (reported elsewhere) ${ }^{13}$ suggests that the slightly earlier advice and treatment provided by PhysioDirect means that the service is likely to be more cost effective than usual pathways of care from a health service perspective, which will be of interest to commissioners.

In future, PhysioDirect services will probably be provided more often in conjunction with direct access for patients to physiotherapists (rather than after referral from another healthcare professional), and PhysioDirect could be offered as a choice for patients wanting quicker advice rather than as the only route to care. ${ }^{29}$ Patients who self refer will probably have less serious problems of shorter duration than those referred by general practitioners, ${ }^{29}$ and in these circumstances, the benefits of initial telephone assessment and advice via PhysioDirect could be greater.

The PhysioDirect services in this study used experienced physiotherapists to provide telephone assessments supported by computerised assessment templates. Some PhysioDirect services that have been recently established use less experienced physiotherapists who are not supported by computerised templates. It should not be assumed that our findings are applicable under these circumstances.

We thank the patients who contributed to this research; the physiotherapists, administrative staff, managers, and commissioners who supported the set up and delivery of the trial in the four primary care trusts; participating general practices; the research support staff in Bristol and Keele; members of the trial steering committee and data monitoring committee; and Jill Gamlin and Nick Deane who developed the PhysioDirect software used in this trial.

Contributors: CS was the chief investigator, and did the drafting and final editing of paper. AAM advised about the trial design and supervised the statistical analysis. SH contributed to the study design, outcome assessment, and analysis. $\mathrm{CH}$ was the trial manager. $\mathrm{AB}$ participated in recruitment, data collection, and coordination in sites near Keele. JC was involved in the study design and analysis. SK was involved in recruitment, data collection, and coordination in sites near Bristol. AF undertook statistical analysis. JH and SG were involved in the design and implementation of PhysioDirect services and the trial. NEF contributed to the trial design and was the principal investigator for the Stoke-on-Trent and Cheshire sites. All authors contributed to the final version of the paper. CS is the guarantor.

Funding: The research was funded by the Medical Research Council (MRC) and managed by the National Institute for Health Research (NIHR) on behalf of the MRC-NIHR partnership. The funder had no role in the study design; the collection, analysis, or interpretation of data; the writing of the report; or the decision to submit the paper for publication. The researchers are all independent of the funders. All researchers had access to all the data.

Competing interests: All authors have completed the Unified Competing Interest form at www.icmje.org/coi_disclosure.pdf (available on request from the corresponding author) and declare: all authors had financial support from the MRC for the submitted work; no financial relationships with any organisations that might have an interest in the submitted work in the previous 3 years; no other relationships or activities that could appear to have influenced the submitted work.

Ethical approval: Multisite research ethics approval was obtained from Southmead research ethics committee, reference 08/H0102/95. All participants gave informed written consent. 


\section{What is already known on this topic}

Under the PhysioDirect care pathway, patients are invited to telephone a physiotherapist for initial assessment and advice, followed by face-to-face physiotherapy if necessary

Several observational studies have suggested benefits of PhysioDirect services for patients with musculoskeletal problems, in relation to improved process of care and patient satisfaction

Research on similar systems of telephone assessment and advice in other clinical settings has shown that they can be safe, clinically accurate, cost effective, and acceptable to patients, and can reduce clinicians' workload

\section{What this study adds}

Half of the patients referred by general practitioners to physiotherapy could be managed by telephone, reducing delays for initial treatment advice and non-attendance rates at subsequent face-to-face appointments

PhysioDirect was as clinically effective as usual care (based on joining waiting lists for appointments without telephone consultation), and seemed to be safe

Reduced delays for physiotherapy were not associated with increased patient satisfaction with access to care, and overall satisfaction of patients was slightly lower for PhysioDirect than for usual care

Data sharing: Participants did not give informed consent for data sharing but the data are anonymised and the risk of identification is low. Data from the trial may be available from the corresponding author at c.salisbury@bristol.ac.uk subject to agreement about the use of the data.

1 Bunn F, Byrne G, Kendall S. Telephone consultation and triage: effects on health care use and patient satisfaction. Cochrane Database Syst Rev 2004;CD004180.

2 Jordan KP, Kadam UT, Hayward R, Porcheret M, Young C, Croft P. Annual consultation prevalence of regional musculoskeletal problems in primary care: an observational study. BMC Musculoskelet Disord 2010;11:144.

3 Brevick H, Collett B, Ventafridda V, Cohen R, Gallacher D. Survey of chronic pain in Europe:prevalence, impact on daily life, and treatment. Eur J Pain 2006;10:287-333.

4 Maniadakis N, Gray A. The economic burden of back pain in the UK. Pain 2000;84:95-103.

5 Health and Social Care Information Centre NHS Physiotherapy Services Summary Information for 2004-2005, England. 2005. https://catalogue.ic.nhs.uk/publications/hospital/ outpatients/nhs-phys-serv-summ-eng-2004-05/nhs-phys-serv-summ-eng-2004-05-rep. pdf.

6 Consulting J. A survey of NHS physiotherapy waiting times and musculoskeletal workload and caseload in England 2010-11. Chartered Society of Physiotherapy, 2011.

7 Miller J, Gross A, D'Sylva J, Burnie SJ, Goldsmith CH, Graham N, et al. Manual therapy and exercise for neck pain: a systematic review. Man Ther 2010;15:334-54

8 Green S, Buchbinder R, Hetrick S. Physiotherapy interventions for shoulder pain. Cochrane Database Syst Rev 2003:CD004258.

9 Conaghan PG, Dickson J, Grant RL. Care and management of osteoarthritis in adults: summary of NICE guidance. BMJ 2008;336:502-3.

10 Taylor S, Ellis I, Gallagher M. Patient satisfaction with a new physiotherapy telephone service for back pain patients. Physiotherapy 2002;88:645-57.

11 Gamlin J. Direct access to physiotherapy. 2005. www.wipp.nhs.uk/db.php?gpuid=1\& type=bro

12 Foster NE, Williams B, Grove S, Gamlin J, Salisbury C. The evidence for and against 'PhysioDirect' telephone assessment and advice services. Physiotherapy 2011;97:78-82.

13 Salisbury C, Foster NE, Hopper C, Bishop A, Hollinghurst S, Coast J, et al. A pragmatic randomised controlled trial of the effectiveness and cost-effectiveness of 'PhysioDirect' telephone assessment and advice services for physiotherapy. Health Technol Assess 2013;17.

14 Salisbury C, Foster NE, Bishop A, Calnan M, Coast J, Hall J, et al. 'PhysioDirect' telephone assessment and advice services for physiotherapy: protocol for a pragmatic randomised controlled trial. BMC Health Serv Res 2009;9:136.

15 Bishop A, Gamlin J, Hall J, Hopper C, Foster NE. PhysioDirect: supporting physiotherapists to deliver telephone assessment and advice services within the context of a randomised trial. Physiotherapy 2012, doi:10.1016/.j.physio.2012.08.002.
16 Ware JE, Kosinski M, Bjorner JB, Turner-Bowker JM, Gandek B, Maruish ME. User's manual for the SF36v2 Health Survey. Quality Metric, 2007.

17 Walsh TL, Hanscom B, Lurie JD, Weinstein JN. Is a condition-specific instrument for patients with low back pain/leg symptoms really necessary? The responsiveness of the Oswestry Disability Index, MODEMS, and the SF-36. Spine 2003:28:607-15.

18 Kvien TK, Kaasa S, Smedstad LM. Performance of the Norwegian SF-36 Health Survey in patients with rheumatoid arthritis. II. A comparison of the SF-36 with disease-specific measures. J Clin Epidemiol 1998;51:1077-86.

19 Angst F, Aeschlimann A, Steiner W, Stucki G. Responsiveness of the WOMAC osteoarthritis index as compared with the SF-36 in patients with osteoarthritis of the legs Lurie J. A review of generic health status measures in patients with low back pain. Spine 2000;25:3125-29.

21 Paterson C. Measuring outcomes in primary care: a patient generated measure, MYMOP, compared with the SF-36 survey. BMJ 1996;312:1016-20.

22 EQ-5D. A standardised instrument for use as a measure of health outcome. 2012. www. euroqol.org/home.html.

23 Pham T, Van Der HD, Lassere M, Altman RD, Anderson JJ, Bellamy N, et al. Outcome variables for osteoarthritis clinical trials: The OMERACT-OARSI set of responder criteria. J Rheumatol 2003;30:1648-54

24 Schulz KF, Grimes DA. Multiplicity in randomised trials I: endpoints and treatments. Lancet 2005;365:1591-5

25 Sterne JAC, White IR, Carlin JB, Spratt M, Royston P, Kenward MG, et al. Multiple imputation for missing data in epidemiological and clinical research: potential and pitfalls. BMJ 2009;338:b2393.

26 Tunis SR, Stryer DB, Clancy CM. Practical clinical trials: increasing the value of clinical research for decision making in clinical and health policy. JAMA 2003;290:1624-32.

27 Black N. Why we need observational studies to evaluate the effectiveness of health care. BMJ 1996;312:1215-8.

28 Holdsworth LK, Webster V. An international multi-centred investigation of patient self referral and physiotherapy practice in privately funded health care systems. 2009. http:// s3.amazonaws.com/zanran_storage/www.selfreferralphysioinfo.com/ContentPages/ 44216519.pdf.

29 Department of Health. Self-referral pilots to musculoskeletal physiotherapy and the implications for improving access to other AHP services. Department of Health, 2008.

Accepted: 19 December 2012

\section{Cite this as: BMJ 2013;346:f43}

This is an open-access article distributed under the terms of the Creative Commons Attribution Non-commercial License, which permits use, distribution, and reproduction in any medium, provided the original work is properly cited, the use is non commercial and is otherwise in compliance with the license. See: http://creativecommons.org/licenses/bync/2.0/ and http://creativecommons.org/licenses/by-nc/2.0/legalcode. 


\section{Tables}

Table 1| Baseline characteristics of randomised patients*

$$
\text { No (\%) of patients }
$$

Usual care $(n=743,33 \%)$ PhysioDirect $(n=1506,67 \%)$

\begin{tabular}{|c|c|c|}
\hline Female sex $\uparrow$ & $438(59)$ & $897(60)$ \\
\hline Age (years) †‡ & $48(36-62)$ & $48(37-61)$ \\
\hline White ethnicity & $705(96)$ & $1,439(97)$ \\
\hline Employed & $417(57)$ & $925(62)$ \\
\hline \multicolumn{3}{|l|}{ Site of musculoskeletal problem $†$} \\
\hline Cervical & $89(12)$ & $185(12)$ \\
\hline Thoracic & $13(2)$ & $35(2)$ \\
\hline Lumbar & $203(27)$ & $412(27)$ \\
\hline Upper limb & $174(23)$ & $351(23)$ \\
\hline Lower limb & $225(30)$ & $450(30)$ \\
\hline Widespread pain & $7(1)$ & $8(1)$ \\
\hline Multiple & $27(4)$ & $55(4)$ \\
\hline Other & $4(1)$ & $10(1)$ \\
\hline \multicolumn{3}{|l|}{ Primary care trust $†$} \\
\hline Bristol & $251(34)$ & $499(33)$ \\
\hline Somerset & $165(22)$ & $348(23)$ \\
\hline Cheshire & $174(23)$ & $353(23)$ \\
\hline Stoke & $153(21)$ & $306(20)$ \\
\hline Time off work (days) $\ddagger$ & $0(0-5)$ & $0(0-5)$ \\
\hline SF-36v2 (physical component score)§ & $37.72(8.63)$ & $36.81(8.88)$ \\
\hline
\end{tabular}

*Excluding seven patients excluded after randomisation.

†Stratification/minimisation variable.

¥Median (interquartile range).

§Mean (standard deviation). 
Table 2| Between group differences in SF-36v2 physical component score

\begin{tabular}{|c|c|c|c|c|}
\hline \multirow[b]{2}{*}{ Time after randomisation } & \multicolumn{2}{|c|}{ Mean (standard deviation) score, sample size } & \multirow[b]{2}{*}{ Difference $(95 \% \mathrm{CI})^{*}$} & \multirow[b]{2}{*}{$\mathbf{P}$} \\
\hline & Usual care & PhysioDirect & & \\
\hline 6 weeks & 41.81 (10.30), 653 & 41.57(10.26), 1332 & $0.42(-0.28$ to 1.12$)$ & 0.24 \\
\hline 6 months $\dagger$ & 44.18 (10.84), 629 & 43.50 (10.94), 1283 & $-0.01(-0.80$ to 0.79$)$ & 0.99 \\
\hline
\end{tabular}

*Adjusted for outcome at baseline, sex, age, referral problem, and primary care trust. †Defined as the primary follow-up point. 


\begin{tabular}{|c|c|c|c|c|}
\hline & \multicolumn{2}{|c|}{ Mean (SD) score, sample size } & \multicolumn{2}{|c|}{ Regression analysis $^{*}$} \\
\hline & Usual care & PhysioDirect & Difference $(95 \% \mathrm{Cl})$ & $\mathbf{P}$ \\
\hline \multicolumn{5}{|c|}{ Measure Yourself Medical Outcomes Profile† } \\
\hline Baseline & $3.80(0.99), 743$ & $3.84(0.99), 1504$ & - & - \\
\hline 6 weeks & $2.92(1.21), 561$ & $2.76(1.28), 1101$ & $-0.19(-0.30$ to -0.07$)$ & 0.001 \\
\hline 6 months & $2.40(1.38), 518$ & $2.40(1.43), 1033$ & $-0.02(-0.16$ to 0.11$)$ & 0.73 \\
\hline \multicolumn{5}{|l|}{ EQ-5D $\ddagger$} \\
\hline Baseline & $0.56(0.29), 731$ & $0.53(0.30), 1480$ & - & - \\
\hline 6 weeks & $0.64(0.26), 554$ & $0.64(0.27), 1080$ & $0.01(-0.01$ to 0.03$)$ & 0.29 \\
\hline 6 months & $0.69(0.27), 508$ & $0.69(0.27), 1020$ & $0.01(-0.02$ to 0.03$)$ & 0.61 \\
\hline \multicolumn{5}{|c|}{ Global improvement score $\ddagger$} \\
\hline Baseline & NA & NA & NA & NA \\
\hline 6 weeks & 3.54 (1.19), 552 & $3.69(1.28), 1089$ & 0.15 (0.02 to 0.28$)$ & 0.02 \\
\hline 6 months & 4.07 (1.40), 501 & $4.01(1.44), 1001$ & $-0.08(-0.23$ to 0.08$)$ & 0.32 \\
\hline \multicolumn{5}{|c|}{ Response to treatment using OMERACT-OARSI criteria ${ }^{23} \neq \S$} \\
\hline & NA & NA & NA & NA \\
\hline 6 weeks & $124(22.6) / 550$ & $306(28.2) / 1085$ & $1.37(1.08$ to 1.75$)$ & 0.01 \\
\hline 6 months & $197(38.6) / 510$ & $430(41.8) / 1029$ & 1.14 (0.92 to 1.43$)$ & 0.24 \\
\hline
\end{tabular}

$\mathrm{NA}=$ not applicable; OMERACT=Outcome Measures in Rheumatology; OARSI=Osteroarthritis Research Society International; SD=standard deviation. *Adjusting for baseline outcome, age, sex, referral problem, and primary care trust. †Lower value indicates better outcome.

$\ddagger$ Higher value indicates better outcome.

$\S$ Data are no (\%) of participants who responded/total sample size, and odds ratio ( $95 \%$ confidence interval). 


\begin{tabular}{|c|c|c|c|c|c|c|c|c|}
\hline \multirow[b]{3}{*}{ Outcome } & \multicolumn{6}{|c|}{ Mean (standard deviation) score, sample size } & \multicolumn{2}{|c|}{$\begin{array}{l}\text { Regression analysis* (arm } \\
\text { difference }(95 \% \mathrm{Cl}), \mathrm{P})\end{array}$} \\
\hline & \multicolumn{2}{|c|}{ Baseline } & \multicolumn{2}{|c|}{6 weeks } & \multicolumn{2}{|c|}{6 months } & \multirow[b]{2}{*}{6 weeks } & \multirow[b]{2}{*}{6 months } \\
\hline & UC & PD & UC & PD & UC & PD & & \\
\hline $\begin{array}{l}\text { Mental } \\
\text { component } \\
\text { score }\end{array}$ & $\begin{array}{c}45.7(13.3) \\
743\end{array}$ & $\begin{array}{c}46.1(12.6) \\
1504\end{array}$ & $\begin{array}{c}48.7(12.2) \\
653\end{array}$ & $\begin{array}{c}48.7(12) \\
1332\end{array}$ & $\begin{array}{c}48.7(11.8) \\
629\end{array}$ & $\begin{array}{c}49(11.9) \\
1283\end{array}$ & $\begin{array}{c}-0.03(-0.88 \text { to } \\
0.82), 0.94\end{array}$ & $\begin{array}{c}0.11(-0.83 \text { to } \\
1.05), 0.82\end{array}$ \\
\hline $\begin{array}{l}\text { Physical } \\
\text { functioning }\end{array}$ & $\begin{array}{c}39.9(10.2) \\
743\end{array}$ & $\begin{array}{l}39.3(10.4) \\
1506\end{array}$ & $\begin{array}{c}43.0(10.8) \\
656\end{array}$ & $\begin{array}{l}43.0(10.9) \\
1338\end{array}$ & $\begin{array}{c}45.2(10.8) \\
632\end{array}$ & $\begin{array}{c}44.7(11.2) \\
1284\end{array}$ & $\begin{array}{l}0.52(-0.19 \text { to } \\
1.23), 0.14\end{array}$ & $\begin{array}{c}0.00(-0.75 \text { to } \\
0.75), 1.00\end{array}$ \\
\hline Role-physical & $\begin{array}{c}37.4(11.2) \\
743\end{array}$ & $\begin{array}{c}36.7(10.9) \\
1502 \\
\end{array}$ & $\begin{array}{c}43.2(11.6) \\
655\end{array}$ & $\begin{array}{c}43.0(11.3) \\
1336\end{array}$ & $\begin{array}{c}45.5(11.5) \\
630 \\
\end{array}$ & $\begin{array}{c}45.0(11.5) \\
1284\end{array}$ & $\begin{array}{c}0.27(-0.60 \text { to } \\
1.15), 0.52\end{array}$ & $\begin{array}{c}-0.08(-1.00 \text { to } \\
0.84), 0.86\end{array}$ \\
\hline Bodily pain & $\begin{array}{l}34.9(8.2) \\
743\end{array}$ & $\begin{array}{c}34.6(8.2) \\
1506\end{array}$ & $\begin{array}{c}41.1(9.6) \\
657\end{array}$ & $\begin{array}{c}40.9(9.9) \\
1338\end{array}$ & $\begin{array}{c}43.2(10.5) \\
632\end{array}$ & $\begin{array}{c}43.1(10.6) \\
1284\end{array}$ & $\begin{array}{c}0.06(-0.68 \text { to } \\
0.80), 0.89\end{array}$ & $\begin{array}{c}0.04(-0.81 \text { to } \\
0.90), 0.93\end{array}$ \\
\hline General health & $\begin{array}{c}45.1(10.5) \\
743\end{array}$ & $\begin{array}{l}44.6(10.7) \\
1506\end{array}$ & $\begin{array}{c}45.9(10.5) \\
657\end{array}$ & $\begin{array}{l}45.3(10.8) \\
1338\end{array}$ & $\begin{array}{c}46.1(10.6) \\
634\end{array}$ & $\begin{array}{c}45.7(11.1) \\
1284\end{array}$ & $\begin{array}{c}-0.07(-0.66 \text { to } \\
0.51), 0.81\end{array}$ & $\begin{array}{c}-0.14(-0.79 \text { to } \\
0.51), 0.66\end{array}$ \\
\hline Vitality & $\begin{array}{c}43.7(10.6) \\
743 \\
\end{array}$ & $\begin{array}{l}43.4(10.7) \\
1506\end{array}$ & $\begin{array}{c}46.3(10.8) \\
655\end{array}$ & $\begin{array}{c}46.3(10.8) \\
1334\end{array}$ & $\begin{array}{c}47.4(10.5) \\
632 \\
\end{array}$ & $\begin{array}{c}47.0(11.1) \\
1283\end{array}$ & $\begin{array}{c}0.35(-0.40 \text { to } \\
1.11), 0.35\end{array}$ & $\begin{array}{c}-0.23(-1.05 \text { to } \\
0.60), 0.59\end{array}$ \\
\hline $\begin{array}{l}\text { Social } \\
\text { functioning }\end{array}$ & $\begin{array}{c}40.4(12.8) \\
743\end{array}$ & $\begin{array}{c}39.8(12.6), \\
1504 \\
\end{array}$ & $\begin{array}{c}45.1(12.2) \\
657\end{array}$ & $\begin{array}{c}45.0(12.4) \\
1338 \\
\end{array}$ & $\begin{array}{c}46.1(12.0), \\
632\end{array}$ & $\begin{array}{c}46.2(12.2), \\
1284 \\
\end{array}$ & $\begin{array}{c}0.25(-0.69 \text { to } \\
1.18), 0.58\end{array}$ & $\begin{array}{c}0.34(-0.61 \text { to } \\
1.30), 0.48\end{array}$ \\
\hline Role-emotional & $\begin{array}{c}42.1(14.6) \\
743\end{array}$ & $\begin{array}{c}42.3(14.3) \\
1498\end{array}$ & $\begin{array}{c}46.6(12.7) \\
655\end{array}$ & $\begin{array}{c}46.2(12.9) \\
1334\end{array}$ & $\begin{array}{c}47.1(12.2) \\
630\end{array}$ & $\begin{array}{c}47.1(12.4) \\
1283 \\
\end{array}$ & $\begin{array}{c}-0.32(-1.24 \text { to } \\
0.59), 0.51\end{array}$ & $\begin{array}{c}-0.06(-1.07 \text { to } \\
0.95), 0.92\end{array}$ \\
\hline Mental health & $\begin{array}{c}45.4(11.7) \\
743\end{array}$ & $\begin{array}{c}45.8(11.4) \\
1506\end{array}$ & $\begin{array}{c}47.9(11.4) \\
655\end{array}$ & $\begin{array}{c}48.2(11.2) \\
1334\end{array}$ & $\begin{array}{c}48.3(11.44) \\
632\end{array}$ & $\begin{array}{c}48.7(11.3) \\
1283\end{array}$ & $\begin{array}{c}0.24(-0.55 \text { to } \\
1.02), 0.57\end{array}$ & $\begin{array}{c}0.18(-0.67 \text { to } \\
1.04), 0.72\end{array}$ \\
\hline
\end{tabular}

$\mathrm{UC}=$ usual care; $\mathrm{PD}=$ PhysioDirect.

*Adjusting for baseline outcome, age, sex, referral problem, and primary care trust. 
Table 5| Between group differences in time lost from work. ${ }^{*}$ Data are mean (standard deviation), median (interquartile range), and sample size, unless stated otherwise

\begin{tabular}{|c|c|c|c|c|}
\hline Reason for time lost from work & Usual care & PhysioDirect & Arm time ratio $(95 \% \mathrm{Cl}) \dagger$ & $\mathbf{P}$ \\
\hline \multicolumn{5}{|l|}{ Condition for which referred (days) } \\
\hline Baseline & 3.10 (7.72), 0 (0-2), 395 & 3.00 (7.53), 0 (0-2), 866 & NA & NA \\
\hline 6 weeks & 5.35 (15.22), 0 (0-2), 253 & 4.39 (11.44), 0 (0-2), 511 & $-0.95(-3.02$ to 1.05$)$ & 0.27 \\
\hline 6 months & 7.14 (22.24), 0 (0-2), 200 & 7.01 (18.03), 0 (0-3), 390 & $0.08(-3.21$ to 3.35$)$ & 0.94 \\
\hline \multicolumn{5}{|l|}{ Physiotherapy (h) } \\
\hline Baseline & NA & NA & NA & NA \\
\hline 6 weeks & $0.40(1.21), 0(0-0), 656$ & $0.40(2.35), 0(0-0), 1340$ & $-0.01(-0.14$ to 0.18$)$ & 0.94 \\
\hline 6 months & 0.98 (2.80), 0 (0 to 0$), 598$ & 0.95 (4.73), $0(0-0), 1211$ & $-0.04(-0.37$ to 0.33$)$ & 0.85 \\
\hline
\end{tabular}

$\mathrm{NA}=$ not applicable.

*Analysis only included patients who were in work.

†Adjusting for baseline outcome, age, sex, referral problem, and primary care trust. 
Table $6 \mid$ Between group differences in patient satisfaction at six week and six month follow-up

\begin{tabular}{|c|c|c|c|c|}
\hline & \multicolumn{2}{|c|}{ Satisfaction (\% of maximum score $($ mean $(\mathrm{SD}))$, sample size) } & \multicolumn{2}{|c|}{ Regression analysis $\dagger$} \\
\hline & Usual care & PhysioDirect & Arm difference $(95 \% \mathrm{Cl})$ & $\mathbf{P}$ \\
\hline \multicolumn{5}{|c|}{ Satisfaction with physiotherapy consultation } \\
\hline 6 weeks & 82.5 (17.6), 378 & 77.6 (18.7), 890 & $-4.9(-7.1$ to -2.7$)$ & $<0.001$ \\
\hline 6 months & 79.2 (19.2), 368 & 75.7 (20.1), 750 & $-3.4(-5.9$ to 0.97$)$ & 0.005 \\
\hline \multicolumn{5}{|c|}{ Satisfaction with access to physiotherapy } \\
\hline 6 weeks & $71.4(21.1), 381$ & 70.6 (22.3), 869 & $-0.8(-3.4$ to 1.7$)$ & 0.502 \\
\hline 6 months & $69.1(20.8), 367$ & $69.2(22.0), 740$ & $0.1(-2.7$ to 2.8$)$ & 0.960 \\
\hline \multicolumn{5}{|c|}{ Overall satisfaction with service } \\
\hline 6 weeks & 83.7 (25.2), 383 & $77.3(27.4), 885$ & $-6.5(-9.6$ to -3.4$)$ & $<0.001$ \\
\hline 6 months & 79.7 (26.5), 367 & 75.9 (28.3), 739 & $-3.8(-7.3$ to -0.3$)$ & 0.031 \\
\hline
\end{tabular}

*Higher scores indicate greater satisfaction.

†Adjusting for baseline outcome, age, sex, referral problem, and primary care trust. 


\section{Figure}

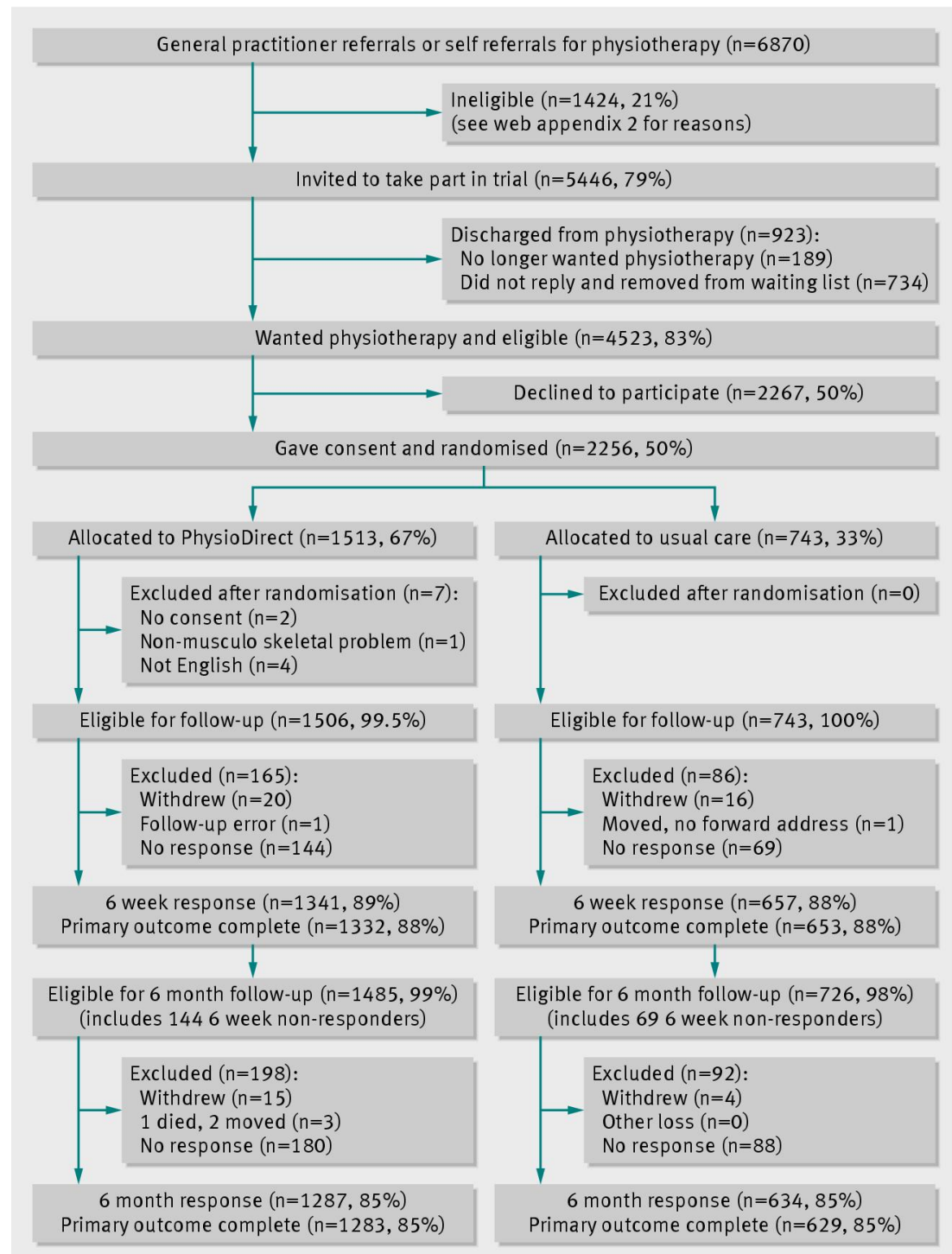

CONSORT diagram: flow of participants through the trial. No English means that patients did not understand English. ${ }^{*}$ Percentages expressed as $n / 1506 \times 100 \%$. †Percentages expressed as $n / 743 \times 100 \%$ 